\title{
Consideraciones sobre el aprendizaje de lenguas extranjeras en situaciones informales y su posible vinculación con el aprendizaje en situaciones formales
}

\section{Ingrid RAMÍREZ DAVID \\ Universidad Nacional Autónoma de México}

\begin{abstract}
El aprendizaje de una lengua extranjera se da tanto en situaciones formales como informales. Aunque los aprendizajes en situaciones formales son buscados y valorizados en nuestra sociedad actual, resulta interesante e importante conocer qué actividades en nuestra vida diaria repercuten en el aprendizaje de otra lengua con el fin de vincularlas con nuestra práctica docente y potenciar la adquisición de la lengua por parte de los alumnos.
\end{abstract}

PALABRAS CLAVE: aprendizaje formal, aprendizaje informal, aprendizaje de lenguas extranjeras, TIC.

The learning of a foreign language occurs in both formal and informal situations. Although learning in formal situations is sought and valued in our current society, it is interesting and important to know what activities that we carry out in our daily life result in the learning of another language in order to link them with our teaching practice and enhance the acquisition of the language by the students.

KEY WORDS: formal learning, informal learning, foreign language learning, ICT.

El gran desarrollo de las tecnologías de la información y de la comunicación (TIC), así como la posibilidad cada vez mayor de viajar y de hacer intercambios comerciales y culturales nos han llevado a tener un mayor contacto con lenguas y culturas distintas a la nuestra. Por lo tanto, tenemos más opciones de aprender otras lenguas o de reforzar nuestro conocimiento de las mismas en situaciones informales. Una de las características primordiales del ser humano es su capacidad para aprender a lo largo de la vida y en distintas circunstancias. Nuestro aprendizaje no se reduce al espacio escolar, sino que aprendemos de manera más o menos consciente de nuestras actividades cotidianas. Hoy por hoy, el requisito de contar con certificados que consten que la persona tiene el conocimiento y las competencias en una lengua determinada, le ha dado un peso excesivo a los aprendizajes de ésta en situaciones formales, es decir, a los aprendizajes que se producen en el seno de una institución reconocida y certificada por instancias oficiales. No obstante, gran parte del aprendizaje de una lengua puede tener lugar fuera de estas instituciones. Pese a que se le adjudique tanto valor a la formalización de los aprendizajes, me parece interesante conocer la frecuencia e importancia de los aprendizajes informales, ya que el ser humano aprende y sigue aprendiendo 
dentro y fuera de los contextos escolares a lo largo de su vida como al realizar actividades laborales o de esparcimiento o al relacionarse con otros individuos. En este escrito reporto parte de la investigación que llevé a cabo para mi trabajo de tesis de la maestría, puntualizando sobre los aspectos que los profesores de lengua debemos considerar en nuestra práctica docente para potenciar los aprendizajes en el aula.

En la actualidad, los sistemas educativos buscan valorar las habilidades comunicativas de cada individuo (no sólo en su lengua materna sino también en distintas lenguas), así como proveer de herramientas para que cada uno pueda desarrollar, de manera autónoma y a lo largo de su vida, habilidades para desempeñarse en nuestra sociedad. La UNESCO y otros organismos internacionales hacen hincapié en el hecho de que la competencia lingüística debe ser plural. El Marco común europeo de referencia para las lenguas (MCERL) asevera, por ejemplo, que:

[...] el enfoque plurilingüe enfatiza el hecho de que conforme se expande la experiencia lingüística de un individuo en los entornos culturales de una lengua, desde el lenguaje familiar hasta el de la sociedad en general, y después hasta las lenguas de otros pueblos (ya sean aprendidas en la escuela o en la universidad, o por experiencia directa), el individuo no guarda estas lenguas y culturas en compartimentos mentales estrictamente separados, sino que desarrolla una competencia comunicativa a la que contribuyen todos los conocimientos y las experiencias lingüísticas y en la que las lenguas se relacionan entre sí e interactúan (Consejo de Europa, 2002: 4).

La importancia de aprender otras lenguas ha dejado de vincularse sólo con la capacidad de adquirir el léxico y la sintaxis de éstas. El aprendizaje de una lengua, hoy en día, se relaciona también con la posibilidad de entender otras formas de ser a través de la exploración de distintas maneras de ver y de concebir el mundo. Las competencias de plurilingüismo y pluriculturalidad se han convertido en puntos clave para una mejor comprensión y respeto de la alteridad. Los individuos, al realizar nuestras actividades cotidianas tales como traslados, compras, trabajo y de esparcimiento, estamos en contacto continuo con otras lenguas y culturas. Este contacto es más fehaciente cuando desempeñamos actividades en línea, tales como participar en redes sociales, ver videos y series, o jugar videojuegos. Así, por ejemplo, a través de las redes sociales nos podemos comunicar con personas de otros países en lenguas distintas de la nuestra; y, de esta manera, desarrollamos la competencia lectora en otro idioma, e incluso podemos llegar a escribir comentarios en la lengua meta y, por ende, practicar la producción escrita. La variedad de materiales a los que tenemos acceso, principalmente en internet, son potenciales fuentes de aprendizajes en situaciones informales, y pueden favorecer los aprendizajes en situaciones formales y viceversa.

Para entender con mayor precisión la noción de aprendizaje informal es necesario revisar las definiciones de los aprendizajes formales y no formales. La trilogía de lo "formal, no formal e informal" fue establecida por organismos internacionales también en los años setenta. Retomaremos aquí lo que Daniel Schugurensky (2007: 14) resumió de estas tres nociones. 
En cuanto a la educación formal, se refiere a la organización institucional (desde el preescolar hasta la universidad) altamente regulada por el Estado que se apega a programas precisos que cuentan con objetivos establecidos, evaluaciones explícitas, manuales seleccionados y aprobados por instancias gubernamentales, y que son impartidos por profesores con las certificaciones solicitadas. La educación formal es altamente jerarquizada donde los ministerios de la educación se encuentran en la cima y los alumnos en la base. Otra característica de este tipo de educación es su naturaleza propedéutica: cada nivel prepara para el siguiente y así los alumnos pueden pasar de uno a otro, para finalmente obtener un certificado o título.

En lo que respecta a la educación no formal, Schugurensky la relaciona con toda actividad educativa organizada que se desarrolla fuera del sistema escolarizado oficial. Generalmente incluye diversos programas con objetivos a corto plazo, no implica prerrequisitos y emplea instructores formados en los temas. Finalmente, la educación informal está constituida por todo lo que no es formal o no formal.

Si bien la mayoría de los autores concuerda con la definición de la educación formal, no es el caso de la educación no formal e informal. Rebeca Mejía (2005b: 5) identifica limitantes en cuanto a las conceptualizaciones de la terminología de aprendizajes informales como el valor "menor o inferior" que se otorga a lo aprendido en situaciones informales. Otra de las limitantes identificados es el hecho de que la caracterización de "formal" o "informal" se aplique tanto a los procesos de aprendizaje como a los escenarios de aprendizaje. En cuanto al aprendizaje informal, los autores coinciden con que éste abarca el aprendizaje que se da fuera de los contextos formales y no formales. Es decir, el que se da fuera de toda institución o actividad con fines de enseñanza. De esta manera, uno puede aprender acerca de una costumbre de otra cultura en una simple conversación. Los autores no se ponen de acuerdo en la definición en términos de la intencionalidad. Schugurensky (2007: 16) distingue tres formas en lo que se refiere al aprendizaje informal, según la presencia de una intencionalidad y de la conciencia que se tenga de estar aprendiendo o de haber aprendido, en un momento dado, al vivir una experiencia. La primera de estas tres formas es el aprendizaje autodirigido, que se caracteriza tanto por la intención de aprender como por la conciencia de que se está aprendiendo. Ésta se relaciona con procesos de aprendizaje autodidactas en distintas circunstancias. La segunda es el aprendizaje fortuito, que se caracteriza por la conciencia de que se ha aprendido pero sin tener la intención de hacerlo. Este fenómeno sucede frecuentemente en situaciones laborales donde se realizan tareas de una manera recurrente y en las que se podría volverse consciente, de pronto, de un conocimiento al comunicarse con un compañero. La tercera forma es la socialización. En este tipo de aprendizaje, la persona no tiene ninguna intención de aprender ni tampoco es consciente de que está aprendiendo. Esto nos sucede en nuestras actividades cotidianas cuando aprendemos sin saberlo de nuestras acciones y relaciones pero de manera no consciente. Como lo asevera Gilles Brougère (2007: 117), el concepto de aprendizaje "informal" parte de una definición negativa que se refiere a lo que no es. De hecho, existe una jerarquía desde la percepción social: en la punta de la pirámide 


\section{$186 \square$ CONSIDERACIONES SOBRE EL APRENDIZAJE DE LENGUAS EXTRANJERAS}

se encuentra la educación formal seguida de la educación no formal y en la base la informal. Esta última se considera como la menos importante de las tres, aunque paradójicamente la mayor cantidad de aprendizajes significativos que se utilizan en la vida diaria provienen de ella (Schugurensky, 2007: 14). Hay que subrayar que antes de entrar a cualquier sistema escolarizado hemos aprendido cosas esenciales para nuestra vida tales como caminar, hablar e interactuar con nuestros semejantes, entre muchas otras.

Para los términos de "aprendizaje formal" y "aprendizaje informal", ampliamente difundidos en la bibliografía, prefiero usar la noción de "situación de aprendizaje formal" (o informal), debido a que, como lo han explicado ya Haydée Silva y Gilles Brougère (2016: 58), no se trata realmente de la formalidad o no del aprendizaje, sino del grado de formalidad de la situación en la que se da.

De acuerdo con Hélène Bézille (2008: 3-4), el proceso histórico de legitimización de los aprendizajes informales ha sido lento y discontinuo; según la autora, éste sigue dos pautas: la del enfoque globalizador de la educación, y la de la visibilidad de las modalidades de aprendizaje que se dan fuera de la forma escolar occidental. Los aprendizajes en situaciones informales han sido por mucho tiempo relegados a un segundo plano o ignorados por los investigadores del ámbito educativo. Sin embargo, como lo subraya Rebeca Mejía (2005a: 2), es a raíz “del reconocimiento de una crisis mundial en la educación formal [que] ha prosperado un enfoque de estudio del aprendizaje contrastando el aprendizaje informal con el formal". En 1926, Lindeman utilizó por primera vez la noción de aprendizaje informal para referirse a la capacitación de los adultos, y mencionaba como "une aventure coopérative d'apprentissage informel, non autoritaire dont le but est de découvrir la signification de l'expérience"1 (Lindeman, 1926, en Brougère y Bézille, 2007: 120). De este modo, el adulto aprende de tareas cotidianas y adquiere cierta experiencia que puede compartir con otros. Asimismo, el adulto es motivado por la necesidad de aprender con un fin específico y, por lo tanto, toma las riendas de su propia formación con el fin de satisfacer dicha carencia cognitiva. Hoy en día, los tutoriales o las Wikis nos permiten aprender de manera inmediata a resolver situaciones de la vida cotidiana, como podría ser el mismo uso de nuestras herramientas tecnológicas. En los años setenta, Allen Tough, investigador canadiense, pionero en estudios empíricos sobre aprendizajes informales, resaltó que el aprendizaje informal solía ser una práctica común entre los adultos aunque resultase ser invisible. Uno de sus hallazgos fue que $20 \%$ de los aprendizajes de los adultos se daba en un medio formal y que "The other 80 percent was informal. We didn't know what to call it. That's when I came up with this idea of the iceberg as a metaphor, because so much of it is invisible"2 (2002: 3). Esta metáfora nos muestra la necesidad de investigar sobre

\footnotetext{
1 “[...] una aventura cooperativa de aprendizaje informal, no autoritaria cuya meta es descubrir el significado de la experiencia”. (Salvo indicación contraria, las traducciones son mías.)

2 "El otro 80 \% era informal. No sabíamos cómo nombrarlo. Así fue cuando surgió la idea del iceberg como metáfora porque gran parte de esto era invisible".
} 
los aprendizajes informales, ya que una gran parte de éstos escapan a la investigación en educación y pueden ser potenciadores de aprendizajes significativos por parte de los aprendientes.

Silva y Brougère (2016: 60) afirman que en las situaciones formales e informales en el aprendizaje de una lengua extranjera han coexistido durante siglos y han seguido una lógica de oposición y de complementariedad según las circunstancias de aprendizaje, por lo que más bien se entrelazan y forman un continuum. Una lengua extranjera se puede aprender tanto en una institución escolar con programas establecidos, en centros especializados de enseñanza de lenguas, con profesores particulares (con formación profesional o no), así como en el desarrollo de actividades diarias (el uso de TIC viajes, entre otros). Por ejemplo, Geoffrey Sockett (2014: 11) menciona que el aprendizaje de lenguas se da en contextos no académicos pero con material producido profesionalmente con fines didácticos como manuales, audios, videos, CD-ROMs y sitios de internet. Todas estas situaciones pueden incluso coexistir mostrando, con ello, que no hay fronteras intransitables entre lo formal y lo informal. Michael Eraut prefiere considerar el aprendizaje como algo continuo y de ahí que se refiera al aprendizaje informal "as learning that comes closer to the informal end tan the formal end of a continuum"3 (Eraut, 2004: 250).

En un contexto crecientemente plurilingüe y en una época donde la comunicación y el contacto con el otro son casi inmediatos y están al alcance de muchos, existen múltiples situaciones que favorecen el aprendizaje de lenguas extranjeras en situaciones informales. Según Mejía (2005b: 5), la investigación del aprendizaje en situaciones informales se ha enfocado sobre todo a tres áreas: lo cotidiano, la difusión científica o cultural y los contextos no escolares. La primera área se refiere a lo que ocurre en el seno de prácticas sociales de la vida cotidiana en distintos grupos culturales. El aprendizaje de una lengua extranjera podría tener lugar gracias al contacto que se tenga con ese idioma en el contexto diario (trabajo, presencia cultural), como el convivir con colegas extranjeros. La segunda área atañe los escenarios con objetivos de difusión de ciencia, cultura u ocio, como en festivales culturales, exposiciones y conciertos, entre otros eventos organizados por otros países. Tales escenarios son propicios al desarrollo de un aprendizaje de una lengua extranjera. La tercer área tiene que ver con contrastar el aprendizaje en contextos escolares con aquel que se adquiere en contextos no escolares. En este caso, se podría comparar el aprendizaje en situación formal de una lengua, con el que se produce al estar en inmersión, en contacto con hablantes en un contexto cotidiano o al realizar estancias en el país donde se habla la lengua meta.

Una lengua extranjera puede tener mayor o menor presencia o impacto en un contexto determinado según las relaciones económicas, políticas o culturales que se establezcan entre los países. Geoffrey Sockett (2014: 4) afirma que se puede medir la

\footnotetext{
3 “[...] como el aprendizaje que se acerca más al extremo informal que al extremo formal de un continuum".
} 
influencia de una lengua y una cultura a partir del impacto que tiene en las actividades cotidianas de otra.

Actualmente, en nuestro tiempo libre, nos abocamos a actividades generalmente relacionadas con los medios de comunicación tales como ver televisión, escuchar música en diversos formatos y navegar en internet. Dichas actividades son una fuente potencial de contacto con otras lenguas y, por lo tanto, podrían traducirse en un aprendizaje de estas últimas. Una gran parte de películas y series de televisión son originarias de Estados Unidos o Canadá, así como muchas de las canciones de moda. Gérard Bonnet (2002: 69), en su estudio sobre competencias en inglés en ocho países (Alemania, Dinamarca, Finlandia, Francia, España, Noruega, Países Bajos y Suecia), demostró que el nivel de comprensión oral del inglés es más bajo en países donde las películas están dobladas, como es el caso de España y de Francia. Así, deducimos que al ver películas en su lengua original se desarrolla la competencia de comprensión oral. En México, las películas en lengua extranjera generalmente están subtituladas, excepto las destinadas a los niños y las de ciertos canales de televisión nacional que están dobladas. Consecuentemente, las personas que ven cine internacional pueden desarrollar competencias de comprensión oral de otros idiomas. Sockett (2014: 39-40) señala que, actualmente, el modo de escuchar música adopta una diversidad de formas ya que se puede hacer a través de servicios como Spotify, ventas en iTunes o simplemente a través de YouTube. Además con la Web 2.0 existe la posibilidad de interactuar con otros usuarios, cada vez de manera más directa al comentar en plataformas como YouTube o Facebook. De este modo, el papel de quién escucha es más activo: selección del contenido musical, mayor control en el modo de escucha y mayor cantidad de recursos para comprender las letras. Lo que muestra: "how learners can subconsciously create learning systems for themselves in unique combinations"4 (2014: 46). Existen otras fuentes, como el aprendizaje de lenguas asistido por computadoras u otros dispositivos, usadas para aprender lenguas en las que la tecnología juega un papel primordial para permitir acceso remoto a materiales de aprendizaje no presenciales. Asimismo, tenemos acceso al conocimiento de otras lenguas mediante la tecnología.

Jugar videojuegos es otra de las actividades que pueden llevar a un aprendizaje.

Cuando videojugamos se hace necesaria la utilización y la activación de estímulos relacionados con los dos hemisferios del cerebro, de manera interactiva y mutuamente reforzada. La emoción, el instinto, la fantasía y el desorden nos ofrecen la oportunidad de razonar, deducir, analizar y sintetizar con rigor. Sólo así podemos llegar a comprender cómo las tradicionales categorías lógicas no son por sí solas capaces de explicar la complejidad de nuestro convulso mundo y su fascinante universo simbólico (Gramigna, González-Fárraco, 2009: 163).

\footnotetext{
4 “[...] cómo los aprendientes pueden de manera subconsciente crear sus propios sistemas de aprendizaje con combinaciones únicas".
} 
Muchos videojuegos comerciales que no están diseñados con propósitos educativos existen en decenas de lenguas y pueden ser jugados en diversos dispositivos (Sykes y Reinhardt, 2013: 4). Éstos ponen al alcance del jugador la posibilidad de desarrollar nuevas competencias en otras lenguas, ya que el sujeto participa en comunidades conformadas por personas de diversas nacionalidades que hablan distintas lenguas (Sykes y Reinhardt, 2013: 6).

Por otro lado, en internet, las búsquedas de información, la lectura, la comunicación a través de redes sociales y la compra de bienes y servicios se pueden realizar también en otras lenguas. En su investigación, Sockett concluye que "it must be stressed that the vast range of affordances of current online technologies allows highly individualized activities in the target language, which can lead to equally individualised outcomes"5 (2014: 61). Los nuevos dispositivos móviles permiten una interacción en todo momento y con respuesta inmediata, lo que lleva a una mayor interacción y alcance de conocimientos y, por supuesto, a una revolución del tipo de intercambios. Tratar de evidenciar la adquisición de la lengua meta en estos contextos se torna complicado con parámetros tradicionales, ya que el material en lengua meta es desconocido y particular a cada individuo (películas, videos, guiones, textos en blogs, noticias, comunicaciones en redes sociales, etcétera). Además, las actividades realizadas resultan complejas de estudiar. Según Sockett (2014: 111), es necesario acercarse desde una perspectiva semasiológica ${ }^{6}$ para analizar las percepciones de las personas sobre sus prácticas y desde un punto de vista onamosiológico ${ }^{7}$ con el fin de entender cómo ocurre el aprendizaje. La adquisición se puede relacionar con procesos de detección de patrones e imitación de éstos; o con el hecho de intentar darle sentido a lo que se está viendo, leyendo o escuchando, e incluso escribiendo o haciendo.

Como lo podemos observar, durante nuestro tiempo libre realizamos actividades relacionadas con las tecnologías de la información y la comunicación. El uso de éstas nos da acceso a una gran diversidad de actividades como informarse, jugar, ver videos o películas, escuchar música y generalmente éstas llevan al contacto con otras lenguas. Los modelos de input y autonomía son observables durante el desempeño de actividades realizadas en línea donde la persona que navega se encuentra en una situación informal de aprendizaje.

En la actualidad, tenemos mayores oportunidades de conocer otros lugares o incluso de vivir en otros sitios. En un mundo globalizado y moderno, se facilitan los viajes y, así, se incrementan las ocasiones de hablar en lengua meta. Si bien el viajar no está al

\footnotetext{
5 “[...] se debe destacar la amplia gama de posibilidades de las tecnologías en línea actuales, que dan lugar a actividades altamente individualizadas en la lengua meta, las cuales también pueden llevar a resultados personalizados".

${ }^{6}$ Proceso llevado a cabo por el receptor al descodificar el mensaje del emisor (relación significantesignificado o palabra- concepto) (Luna et al., 2005).

${ }^{7}$ Proceso llevado a cabo por el emisor al codificar su mensaje (relación significado-significante o concepto- palabra) (Luna et al., 2005).
} 
alcance de todos, existe la posibilidad de tener contacto con hablantes nativos de lenguas extranjeras por las inmigraciones a nuestro lugar de residencia.

$\mathrm{Al}$ estar en un medio en el que se habla en forma común la lengua meta, el aprendiente está expuesto a ésta un tiempo generalmente mayor que el de una clase de lengua y, por lo tanto, tiene una mayor cantidad de input. María Juan Garau (2008: 59) afirma que "la concentración de tiempo que los aprendices disfrutan en la comunidad de habla de la lengua meta potencialmente puede conducir a ganancias lingüísticas significativas y genera en consecuencia altas expectativas". La estrategia comunicativa implica, por lo tanto, no sólo el input, sino que la construcción de un significado de éste así como la manera de lograr comunicación. En inmersión, el enfoque se centra en la lengua meta y el aprendizaje se da en situaciones comunicativas verdaderas. Acerca de los principios teóricos de la metodología de inmersión, Josu Sierra (1994: 85) menciona los siguientes elementos: exposición temprana e intensiva a la lengua meta; construcción de un "entorno imitativo natural"; interferencia y transferencia entre lenguas; enseñanza contextualizada y estrategia comunicativa. El "entorno imitativo natural" que se trata de crear en metodologías de inmersión se refiere al hecho de exponer al individuo a la lengua no sólo a un mayor tiempo, sino también a elementos culturales, ya que lengua y cultura son indisociables. Al estar continuamente expuestos a la lengua extranjera, el proceso de adquisición se parece al de la lengua materna: se entiende antes de hablar y es un proceso de ensayo y error. En el caso de un viaje o de intercambios con extranjeros, nos exponemos a situaciones y contextos distintos de los habituales y conocemos otras formas de hacer, pensar y vivir circunstancias.

Juan Garau asevera que el aprendizaje de lenguas extranjeras en contextos naturales "permite a los aprendices verificar las hipótesis que formulan acerca del funcionamiento de la lengua meta, practicar ampliamente sus destrezas, cosa que facilita la automatización del conocimiento" (2008: 51). Al estar en inmersión el aprendiente de una lengua recibe retroalimentación positiva o negativa sobre su conocimiento lingüístico al interactuar directamente con los hablantes puesto que, de manera inmediata, sabe si logró comunicar lo que quería según la respuesta del otro. Asimismo, su reacción al mensaje del otro le permite valorar su nivel de comprensión.

En el aula, los alumnos no siempre aprenden o, mejor dicho, no siempre aprenden lo que se supone deberían aprender según los objetivos de la clase de lengua. El aprendizaje es un fenómeno multifactorial pero también individual donde la responsabilidad recae en diversos actores. Varios factores ambientales y contextuales influyen en el aprendizaje desde el contexto social, familiar y escolar.

Desde mediados del siglo pasado, se reconoce casi por consenso que los alumnos deben tener el papel central dentro del proceso de aprendizaje y son la razón de ser de los docentes. Lo que lleva a los jóvenes a aprender o no aprender está relacionado con su situación personal, su nivel socioeconómico, su cultura, sus conocimientos del mundo, sus habilidades propias, sus procesos de aprendizaje, sus expectativas de la materia, sus perspectivas del aprendizaje y lo que esperan del profesor. Los primeros puntos no son modificables; pero el docente sí puede influir y ayudar a los alumnos en 
sus procesos cognitivos si adapta su práctica considerando su situación y sus formas de aprender.

Los contextos formales son percibidos por los alumnos como alejados de su realidad ya que a menudo carecen de "una discusión conceptual sobre cómo integrar analíticamente sus necesidades de distinto tipo, sus capacidades intelectuales, sus objetivos al estar en la escuela, las relaciones que establecen entre contextos sociales (urbanos y rurales) y sus proyecciones hacia el presente y el futuro, entre otros aspectos" (Crovi, López, 2011: 5).

Para este objetivo es importante conocer los intereses de los alumnos, sus gustos, sus actividades fuera de clases y orientar actividades a partir de esta información: "Porque a ellos se dirigen los procesos de enseñanza aprendizaje y de formación como individuos escolarizados. No conocer sus preocupaciones, sus maneras de apropiarse lo que se les enseña o sus formas de vivir en los contextos escolares tiene serias implicaciones para el diseño de los planes de estudio y para la organización de la propia escuela" (5).

En realidad, no existe un aprendizaje formal y uno informal sino que es preferible hablar de un continuum que va de la formalidad a la informalidad en las situaciones de aprendizaje, en función de los contextos en el que dicho aprendizaje se da (Silva, Brougère, 2016: 58). El objetivo que deben plantearse los maestros no es formalizar lo informal ni tratar de "dictar" tareas para que los alumnos las realicen fuera de clase, sino despertar el interés y la curiosidad con respecto al aprendizaje de la otra lengua e incidir en ciertas prácticas para que el alumno siga aprendiendo fuera del contexto escolar como una competencia más para aprender durante toda su vida. Por ejemplo, saber hacer búsquedas en internet, determinar si la información es confiable, actual, y recurrir a textos en su lengua original.

Un punto significativo mencionado por varios autores está relacionado con el uso de las tecnologías de la información y comunicación (TIC) ya que se han vuelto de uso cotidiano:

[...] analizar al estudiante como sujeto social, como joven que responde a los parámetros de una generación que ha desarrollado nuevas estructuras de pensamiento, producto de una cultura digital, en la cual, entre otras muchas cosas, se identifican novedosas formas de aprendizaje, de lectura, de creación del conocimiento y de modos de trabajar. Este conocimiento es indispensable para el rediseño y actualización de los planes de estudio y espacios de formación educativa [...], pero también es fundamental para visualizar las prácticas cotidianas de los jóvenes en materia de entretenimiento, trabajo, relaciones familiares y sociales, mediadas por tecnologías digitales (Crovi, López, 2012: 9).

Las TIC permiten extender el espacio áulico. Conocer al alumno permite mantenerlo interesado en la materia y proponer tareas significativas. Los alumnos suelen tener un mayor dominio del uso de las TIC que muchos docentes, por lo que el intercambio de roles en este ámbito puede aumentar la autoestima del alumno y por lo tanto su 


\section{$192 \square$ CONSIDERACIONES SOBRE EL APRENDIZAJE DE LENGUAS EXTRANJERAS}

motivación. El profesor puede, consecuentemente, trabajar a distancia mediante el uso de redes sociales proponiendo la participación de todos a través de sus propias propuestas de sitios, videos y juegos previamente analizados por él y solicitando, a su vez, que los alumnos compartan sus propios descubrimientos. De este modo se promueve un aprendizaje colaborativo fuera del contexto formal, se fomenta la comunicación entre pares y se desarrolla el sentimiento de pertenencia. Las redes sociales pueden convertirse en un punto de encuentro fundamental para dichos intercambios.

Los hallazgos de los alumnos, socializados ya sea en el aula o fuera de ésta mediante las redes sociales, permiten valorar todo tipo de aprendizaje y, por lo tanto, el aprendizaje en contexto informal deja de ser relegado a segundo plano.

"La valorisation des apprentissages informels permettrait aux individus de quitter ce qui leur est connu et de se lancer dans la découverte et la construction de situations et connaissances nouvelles" (Peter, 2011). ${ }^{8}$

De hecho, abre espacio para hacer nuevas búsquedas $\mathrm{y}$, por lo tanto, para encontrar cosas nuevas que se pueden aprender desde procesos metacognitivos como saberes declarativos, procedimentales o actitudinales. De este modo, el profesor podría recomendar a sus alumnos poner sus dispositivos móviles en la lengua meta. También se puede exhortar a ver películas con subtítulos en la lengua meta, y jugar sus videojuegos cambiando el idioma ya que éstas son actividades que solemos realizar en nuestros tiempos libres.

Otra manera de favorecer el aprendizaje de una lengua en situaciones informales por medio de redes sociales es el intercambio de videos y sitios de interés. Se podría abrir un grupo cerrado en Facebook, Twitter, Whatsapp o similares en el que se compartan videos, canciones, sitios con noticias sobre sus artistas favoritos, información de su interés o incluso sitios o aplicaciones para aprender la lengua meta. Tras fomentar que den su opinión, es importante que el profesor no juegue un rol directivo sino el de un participante más. Aquí se favorece la colaboración y el sentido de pertenencia.

Conforme los alumnos vayan desarrollando sus competencias lingüísticas en la lengua meta se pueden desarrollar materiales más complejos como juegos de preguntas y respuestas sobre cultura general u otros que consistan en la creación de cuentos. Al apelar a su creatividad y al trabajo en equipo se estimula, en efecto, a los alumnos. Estas actividades pueden completarse con materiales digitales, tales como Quizlet, Brainscape y otros similares.

La articulación entre los aprendizajes en situaciones formales y situaciones informales es un tema de estudio relativamente inexplorado en el campo educativo. Conocer los puentes que vinculan los aprendizajes en distintas situaciones permitiría, por un lado, motivar a los aprendientes y fomentar su papel activo en el proceso de aprendizaje y, por el otro, desde el punto de vista institucional, reconsiderar contenidos para elaborar propuestas que sean de interés para los alumnos. Si bien los saberes declarativos, procedimentales y actitudinales relacionados con una lengua suelen adquirirse

8 "La valoración de los aprendizajes informales permitiría a los individuos alejarse de lo que conocen para emprender en el descubrimiento y la construcción de situaciones nuevas". 
en el marco de un aprendizaje en situación formal (salones de clase, escuelas de idiomas), los aprendizajes pueden asimismo obtenerse en situaciones informales de diversa índole (actividades de esparcimiento y juegos), con un peso cualitativo de estos últimos que no resulta desdeñable. Al conocer las prácticas de los alumnos que los llevan a un aprendizaje de lenguas en situaciones informales, los profesores pueden fomentarlas, reforzarlas y acompañar a los aprendientes en el proceso de aprendizaje desde lo formal.

\section{Obras citadas}

BEZILLE, HÉLÈNE. 2008. "De l'apprentissage informel à l'autoformation dans l'éducation tout au long de la vie". L'éducation au long de la vie. Coords. Lucette Colin y Jean-Louis LE GRAND. París: Anthropos. Web: 9 de diciembre de 2017. <http://cma-lifelonglearning.org/frenchbis/pdf/H\%20Bezille $\% 20 I N F O R M E L \% 20$ ET\%20AUTOFORMATIONLIVRECOL.pdf $>$.

BONNET, Gérard. 2002. The assessment of puplils'skills in english in eight european countries. Web: 9 de diciembre de 2017. <https://www.mecd.gob.es/inee/ dam/jcr:d426c134-5c58-44f0-8641-3b5e4354ed37/habilidadesingles2002-1. pdf $>$.

Brougere, Gilles y Hélène BeziLle. 2007. "De l'usage de la notion d'informel dans le champ de l'éducation”. Revue française de pédagogie, núm. 58. Pp. 117-160.

CONSEJO DE EUROPA. 2002. Marco común europeo de referencia para las lenguas. Madrid: Instituto Cervantes. (Versión electrónica.)

Crovi, Delia y Rocío LóPEZ. 2011. “Tejiendo voces. Jóvenes universitarios opinan sobre la apropiación del internet en la vida académica”. Revista Mexicana de Ciencias Sociales, vol. 56, núm. 212. Web: 9 de diciembre de 2017. <http:// www.revistas.unam.mx/index.php/rmcpys/article/view/30421>.

ERAUT, Michael. 2004. Informal learning at the workplace. Studies in Continuing Education, vol. 26, núm. 2. Pp. 247-273. Web: 9 de diciembre de 2017. <http: //www.tandfonline.com/doi/pdf/10.1080/158037042000225245>.

Gramigna, Anita y Juan Carlos GonzÁlez-FARACO. 2009. "Videojugando se aprende: renovar la teoría del conocimiento y la educación”. Comunicar, vol. 17, núm. 33. Pp. 157-164.

JuAn GaraU, María. 2008. Contexto y contacto en el aprendizaje de lenguas extranjeras. Revista Electrònica d'Investigació i Innovació Educativa i Socioeducativa, vol. 1. Pp. 47-66. Web: 28 de febrero de 2017. <http://www.in.uib. cat/pags/volumenes/vol1_num0/maria_garau/index.html $>$.

MEJÍA, Rebeca. 2005. “Aprendizaje informal”. Sinéctica, Revista Electrónica de Educación. Web: 29 de diciembre de 2017. <http://www.redalyc.org/articulo. oa? $\mathrm{id}=99815914001>$. 
. 2005. "Tendencias actuales en la investigación del aprendizaje informal". Sinéctica, Revista Electrónica de Educación. Web: 29 de diciembre de 2017. $<$ http://www.uacm.kirj.redalyc.redalyc.org/articulo.oa?id=99815914002>.

PETER, Jean Michel. 2011. “'Les apprentissages informels', un concept à l'épreuve de la recherche en formation: les enjeux du débat". Savoirs, [en línea], 2011/2, núm. 26, L’Harmattan. Pp. 123-133. <file://C:/Users/Italiano/Downloads/ SAVO_026_0123pdf>.

SCHUGURENSKY, Daniel. 2007. "Vingt mille lieues sous les mers: les quatre défis de l'apprentissage informel". Revue française de pédagogie, núm. 160. Pp. 13-27. Web: 3 de marzo de 2017. <http://rfp.revues.org/583>.

SIERRA, Josu. 1994. "Metodología y práctica en programas de inmersión". Comunicación, lenguaje y educación, 22. Pp. 85-95. Web. <https://dialnet.unirioja. es/descarga/articulo/2941307.pdf>.

SiLVA, Haydée y Gilles BROUGĖRE. 2016. "Jeu entre situations formelles et informelles d'apprentissage des langues étrangères". Synergies Mexique, núm. 6. Pp. 57-68.

SocketT, Geoffrey. 2014. The Online Informal Learning of English. Nueva York: Palgrave Macmillan.

Sykes, Julie y Jonathon ReINHARDT. 2013. Language at Play. Digital Games in Second and Foreign Language Teaching and Learning. Nueva York: Pearson.

TougH, Allen. 2002. "The Iceberg of Informal Adult Learning: New Approaches to Lifelong Learning". Working Paper, núm. 49. Web: 10 de diciembre de 2017. <https://nall.oise.utoronto.ca/res/49AllenTough.pdf $>$. 